Original article

\title{
Antioxidant activity of rat liver with a low resistance to hypoxia after systemic ischemia reperfusion
}

\author{
Gulnar A. Bayburina ${ }^{1}$, Elena A. Nurgaleeva ${ }^{1}$, Ajgul F. Samigullina ${ }^{1}$, Ekaterina R. Farshatova ${ }^{1}$, Timur I. Ganeev ${ }^{1}$, \\ Eduard F. Agletdinov ${ }^{1}$, Tatyana V. Tarasova ${ }^{2}$ \\ ${ }^{1}$ Bashkir State Medical University, Ufa, Russia \\ 2 Ogarev State University of Mordovia, Saransk, Russia
}

Received 28 April 2021, Revised 17 August 2021, Accepted 4 November 2021

C 2021, Russian Open Medical Journal

\begin{abstract}
Objective - To assess the antioxidant activity of rat liver after systemic ischemia reperfusion (IRP).
Material and Methods - The study was conducted on 70 male rats. For all animals of the treatment group $(n=35)$ under ether anesthesia, we were stopping stopping systemic circulation for five minutes. After that, the animals were given an external cardiac massage and artificial lung ventilation. We did not perform circulatory arrest after ether anesthesia in animals of the control group ( $n=35)$. In all animals, we were measuring the levels of serum hormones (corticosterone, aldosterone), the content of glucocorticoid and mineralocorticoid receptors in liver homogenates, and the activity of enzymes of the antioxidant system (superoxide dismutase and catalase). We were making control measurements on days $1,3,5,7,14,21$, and 35 after the simulated IRP.

Results - On day 1 after simulation of IRP development, the levels of cortisol and aldosterone in the serum of treatment group rats were significantly higher, by $14.3 \%$ and $33.5 \%$, respectively, compared with the control group. In response to stress (IRP), we observed the highest concentration of cortisol in the blood of treatment group rats on day 3 ( $p=0.0002)$, which decreased afterwards. On day 1 after IRP, there was a reduction in the activity of superoxide dismutase and catalase in treatment group rats, by $50.3 \%$ and by $29 \%$, respectively $(p<0.0001)$. The lowest antioxidant activity in the rat liver after IRP was observed on days 3-7.

Conclusion - Systemic IRP is associated with pronounced changes in the dynamics of corticosteroid receptors in the liver, which leads to a reduction in the activity of key antioxidant enzymes.
\end{abstract}

Keywords: ischemia-reperfusion injury, corticosteroids, oxidative stress, superoxide dismutase, catalase.

Cite as Bayburina GA, Nurgaleeva EA, Samigullina AF, Farshatova ER, Ganeev TI, Agletdinov EF, Tarasova TV. Antioxidant activity of rat liver with a low resistance to hypoxia after systemic ischemia reperfusion. Russian Open Medical Journal 2021; 10: e0427.

Correspondence to Tatyana V. Tarasova. Phone: +79093280788. E-mail: 9023060@mail.ru.

\section{Introduction}

Ischemia of a post-reperfusion organ is a common cause of its functional insufficiency development, which may even result in death. Ischemia-reperfusion injury often occurs after surgical interventions. Accordingly, in the contemporary literature, cases of ischemia-reperfusion damage to the liver are described, which is often observed when performing operations in the hepatobiliary region using the Pringle maneuver [1]. The latter presumes temporary compression of the hepatoduodenal ligament blood vessels [2], followed by dissection of the liver parenchyma within the ischemic zone (5-10 mm from the demarcation line). This tactic is used during the surgeries, such as liver resection, and in the course of organ transplantation. However, perpetrating even temporary local liver ischemia may result in a significant risk of organ damage due to the IRP development [3].

Some cases of IRP development in the liver after the hemorrhagic shock are known as well [4]. Hemorrhagic shock is a dangerous condition developing due to extensive bleeding. Even if resuscitation efforts with hemorrhagic shock were effective, the patient often has IRP of vital tissues and organs [4]. With systemic IRP, hormones and numerous proinflammatory mediators are released, which leads to the multiple organ failure [5]. With IRP, a large amount of reactive oxygen species is formed, which aggravate reperfusion damage to tissues. Free oxygen radicals cause the oxidation of cell membrane phospholipids, thereby further increasing cell damage [6]. Consequently, systemic ischemia leads to a cascade of pathological reactions, starting with a tissue hypoxia, cell apoptosis, and an increase in the content of reactive oxygen species.

However, despite the fact that the key stages of the local and systemic IRP pathogeneses are known, there are many ambiguous issues regarding the correction of this condition. The IRP development often impedes performing liver resection and transplantation [7]. The state of the post-IRP hepatocyte antioxidant system has not been fully studied as yet.

Objective. To evaluate the liver antioxidant activity in rats with a low resistance to hypoxia after the systemic IRP.

\section{Material and Methods}

The study was performed on 70 sexually mature male white rats. All animals included in the study were not resistant to hypoxia. To comply with this inclusion criterion, we determined 
the resistance of each rat to acute hypobaric hypoxia: during the simulated 'ascent' of a pressure chamber to an 'elevation' of 9000 $\mathrm{m}$, which constituted the threshold for rats with ailments, we identified animals with the greatest inhibition of their motor activity and postural reflexes [5].

Next, under ether anesthesia, we squeezed the vascular bundle of the heart in the treatment group animals $(n=35)$, thereby simulating a five-minute arrest of systemic circulation. Then, these rats were given an external cardiac massage and artificial lung ventilation. We did not perform circulatory arrest after ether anesthesia in animals of the control group $(n=35)$.

All animals were tested for plasma hormones content (corticosterone, aldosterone), the content of glucocorticoid and mineralocorticoid receptors in liver homogenates, and the activity of antioxidant system enzymes (superoxide dismutase and catalase). We performed control measurements on days 1, 3, 5, 7, 14, 21, 35 after the simulated IRP.

The levels of corticosterone and aldosterone in the blood serum was investigated via the immunoradiometric method, using standard test systems from IMMUNOTECH (France). The densities of glucocorticoid and mineralocorticoid receptors in liver homogenates were determined by enzyme-linked immunosorbent assay (ELISA) on the StatFox 2100 analyzer (USA).
We were evaluating liver antioxidant activity via measuring the activity levels of superoxide dismutase and catalase by the ELISA method on the StatFox 2100 analyzer (USA), using standard RANSOD test systems from Randox Laboratories Ltd. (Great Britain)

When performing all manipulations, we complied with the ethical principles of the European Convention for the Protection of Vertebrate Animals Used for Experimental and Other Scientific Purposes (Strasbourg, 1986). The study protocol was approved by the local Ethics Committee at Bashkir State Medical University of the Russian Federation Ministry of Healthcare. In our study, all animals were kept in similar conditions: 6-7 rats per cage, natural lighting, air temperature of $20-22{ }^{\circ} \mathrm{C}$, and unlimited access to water and food.

Statistical processing of our data was conducted using Statistica 8.0 software. Descriptive statistics included calculation of means (medians), standard deviations (interquartile ranges via indicating lower and upper quartiles). For categorical variables, the results were also presented as numbers and percentages. The nonparametric Kruskal-Wallis (H) and Mann-Whitney (U) criteria were used for intergroup comparisons. The differences were considered statistically significant at $p \leq 0.05$.

Table 1. Serum hormone levels in rats with a low resistance to hypoxia in the post-resuscitation period, $M \pm \sigma$

\begin{tabular}{|c|c|c|c|c|c|}
\hline \multirow{2}{*}{ Days } & \multicolumn{2}{|c|}{ Corticosterone, $\mathrm{nmol} / \mathrm{L}$} & \multicolumn{3}{|c|}{ Aldosterone, $\mathrm{pg} / \mathrm{mL}$} \\
\hline & Treatment group & Control group & Treatment group & & Control group \\
\hline \multirow{2}{*}{ Day 1} & $232.35 \pm 54.62$ & $199.12 \pm 40.08$ & $466.13 \pm 115.63$ & & $310.20 \pm 82.70$ \\
\hline & \multicolumn{2}{|c|}{$p=0.0493$} & \multicolumn{3}{|c|}{$p=0.0023$} \\
\hline \multirow{2}{*}{ Day 3} & $334.37 \pm 43.18$ & $181.10 \pm 32.01$ & $453.26 \pm 83.05$ & & $307.17 \pm 79.40$ \\
\hline & \multicolumn{2}{|c|}{$p=0.0002$} & \multicolumn{3}{|c|}{$p=0.0025$} \\
\hline \multirow{2}{*}{ Day 5} & $154.23 \pm 29.10$ & $183.09 \pm 41.2$ & $399.19 \pm 43.03$ & & $308.22 \pm 80.56$ \\
\hline & \multicolumn{2}{|c|}{$p=0.0126$} & \multicolumn{3}{|c|}{$p=0.0032$} \\
\hline \multirow{2}{*}{ Day 7} & $154.16 \pm 24.40$ & $192.11 \pm 38.05$ & $395.60 \pm 65.87$ & & $308.91 \pm 64.60$ \\
\hline & \multicolumn{2}{|c|}{$p=0.0126$} & \multicolumn{3}{|c|}{$p=0.0342$} \\
\hline \multirow{2}{*}{ Day 14} & $127.75 \pm 27.44$ & $195.09 \pm 39.02$ & $402.50 \pm 65.55$ & \multirow{2}{*}{\multicolumn{2}{|c|}{$300.35 \pm 78.80$}} \\
\hline & \multicolumn{2}{|c|}{$p=0.0004$} & & & \\
\hline \multirow{2}{*}{ Day 21} & $189.05 \pm 27.00$ & $201.1 \pm 36.04$ & $466.82 \pm 95.92$ & \multirow{2}{*}{\multicolumn{2}{|c|}{$311.21 \pm 81.50$}} \\
\hline & \multicolumn{2}{|c|}{$p=0.8206$} & & & \\
\hline \multirow{2}{*}{ Day 35} & $223.36 \pm 28.79$ & $198.06 \pm 41.02$ & $473.63 \pm 54.79$ & \multirow{2}{*}{\multicolumn{2}{|c|}{$p=0.0036$}} \\
\hline & \multicolumn{2}{|c|}{$p=0.0494$} & & & \\
\hline
\end{tabular}

Table 2. Dynamics of the corticosteroid receptor content in the liver of rats unresistant to hypoxia in post-resuscitation period, $M \pm \sigma$

\begin{tabular}{|c|c|c|c|c|}
\hline \multirow{2}{*}{ Days } & \multicolumn{2}{|c|}{ GR, mcg per $g$ of protein; $\%$ in relation to the control group } & \multicolumn{2}{|c|}{$\mathrm{MR}, \mathrm{ng}$ per $\mathrm{g}$ of protein; $\%$ in relation to the control group } \\
\hline & Treatment group & Control group & Treatment group & Control group \\
\hline \multirow{2}{*}{ Day 1} & $34.61 \pm 4.30$ & $30.10 \pm 6.45$ & $81.23 \pm 9.21$ & $117.36 \pm 5.06$ \\
\hline & \multicolumn{2}{|c|}{$p=0.0494$} & \multicolumn{2}{|c|}{$p=0.0012$} \\
\hline \multirow{2}{*}{ Day 3} & $29.20 \pm 7.08$ & $30.08 \pm 6.12$ & $104.50 \pm 9.16$ & $118.09 \pm 4.01$ \\
\hline & \multicolumn{2}{|c|}{$p=0.4963$} & \multicolumn{2}{|c|}{$p=0.0125$} \\
\hline \multirow{2}{*}{ Day 5} & $28.15 \pm 6.98$ & $28.87 \pm 5.76$ & $139.50 \pm 10.01$ & $118.44 \pm 5.02$ \\
\hline & \multicolumn{2}{|c|}{$p=0.5233$} & \multicolumn{2}{|c|}{$p=0.0002$} \\
\hline \multirow{2}{*}{ Day 7} & $25.88 \pm 6.01$ & $26.53 \pm 5.91$ & $129.19 \pm 10.82$ & $118.31 \pm 4.63$ \\
\hline & \multicolumn{2}{|c|}{$p=0.1306$} & \multicolumn{2}{|c|}{$p=0.0065$} \\
\hline \multirow{2}{*}{ Day 14} & $22.86 \pm 4.47$ & $23.32 \pm 5.04$ & $150.21 \pm 27.55$ & $117.83 \pm 4.50$ \\
\hline & \multicolumn{2}{|c|}{$p=0.1156$} & \multicolumn{2}{|c|}{$p=0.0052$} \\
\hline \multirow{2}{*}{ Day 21} & $26.49 \pm 9.68$ & $27.15 \pm 7.34$ & $163.12 \pm 10.82$ & $117.62 \pm 4.31$ \\
\hline & \multicolumn{2}{|c|}{$p=0.2988$} & \multicolumn{2}{|c|}{$p=0.0012$} \\
\hline \multirow{2}{*}{ Day 35} & $32.52 \pm 12.86$ & $31.09 \pm 8.41$ & $187.76 \pm 15.16$ & $117.54 \pm 4.22$ \\
\hline & \multicolumn{2}{|c|}{$p=0,6967$} & \multicolumn{2}{|c|}{$p=0,0002$} \\
\hline
\end{tabular}

$\mathrm{MR}$, mineralocorticoid receptors; GR, glucocorticoid receptors. 
Table 3. Dynamic indicators of the antioxidant system activity in the rat liver of both groups

\begin{tabular}{|c|c|c|c|c|}
\hline \multirow{2}{*}{ Monitoring period } & \multicolumn{2}{|c|}{ Superoxide dismutase, units per mg of protein } & \multicolumn{2}{|c|}{ Catalase, $\mathrm{mmol} / \mathrm{min}$ per $\mathrm{mg}$ of protein } \\
\hline & Treatment group & Control group & Treatment group & Control group \\
\hline \multirow{2}{*}{ Day 1} & $14.84 \pm 3.50$ & $29.85 \pm 6.95$ & $5.28 \pm 1.44$ & $7,43 \pm 1,98$ \\
\hline & \multicolumn{2}{|c|}{$p<0.0001$} & \multicolumn{2}{|c|}{$p<0.0001$} \\
\hline Day 3 & \multicolumn{2}{|c|}{$p<0.0001$} & \multicolumn{2}{|c|}{$p<0.0001$} \\
\hline \multirow{2}{*}{ Day 5} & $13.92 \pm 3.90$ & $29.16 \pm 6.88$ & $5.40 \pm 1.40$ & $7,15 \pm 1,92$ \\
\hline & \multicolumn{2}{|c|}{$\mathrm{p}<0.0001$} & \multicolumn{2}{|c|}{$p<0.0001$} \\
\hline Day 7 & \multicolumn{2}{|c|}{$\mathrm{p}<0.0001$} & \multicolumn{2}{|c|}{$p=0.0016$} \\
\hline \multirow{2}{*}{ Day 14} & $18.30 \pm 4.87$ & $29.75 \pm 5.81$ & $6.87 \pm 1.71$ & $7,22 \pm 2,03$ \\
\hline & \multicolumn{2}{|c|}{$p<0.0001$} & \multicolumn{2}{|c|}{$p=0.5988$} \\
\hline \multirow{2}{*}{ Day 21} & $17.70 \pm 3.67$ & $30.04 \pm 5.92$ & $5.67 \pm 1.39$ & $7,31 \pm 2,15$ \\
\hline & \multicolumn{2}{|c|}{$\mathrm{p}<0.0001$} & \multicolumn{2}{|c|}{$p=0.0002$} \\
\hline \multirow{2}{*}{ Day 35} & $23.01 \pm 5.55$ & $29.85 \pm 6.95$ & $7.30 \pm 1.99$ & $7,39 \pm 2,09$ \\
\hline & \multicolumn{2}{|c|}{$p<0.0001$} & \multicolumn{2}{|c|}{$p=0.9998$} \\
\hline
\end{tabular}

\section{Results}

We presented the blood plasma dynamics of corticosterone and aldosterone levels in rats during early and late postresuscitation periods in Table 1. On day 1 after the IRP simulation, the levels of cortisol and aldosterone in the blood serum of the treatment group rats were significantly higher, by $14.3 \%$ and $33.5 \%$, respectively, compared with the control group. In response to stress (IRP), we observed the highest concentration of corticosterone in the blood of treatment group rats on day 3 $(p=0.0002)$, which gradually decreased afterwards and virtually reached the initial levels.

Also, from day 3 on, we have been observing a gradual increase in the blood level of aldosterone in treatment group rats. From day 5 on, we have been detecting a distinct predominance of mineralocorticoid concentration over glucocorticoids.

The dynamics of the corticosteroid receptor content in the liver of rats unresistant to hypoxia in the post-resuscitation period is shown in Table 2. The maximum density of glucocorticoid receptors was established in the treatment group rats on day 1 after the IRP ( $p=0.0494)$, while the minimum density was detected on day 14. On all other remaining control measurement days, the values of this indicator in both groups (treatment and control) were similar.

The content of mineralocorticoid receptors in the treatment group rats significantly differed throughout the entire observation period (Table 2). On day 1, we recorded the smallest number of receptors, whereas from day 3 on, there was a gradual increase in this indicator, and from day 5 on, mineralocorticoid receptors explicitly prevailed, in terms of their numbers, over glucocorticoid receptors.

Over the period, encompassing days 14 through 35, we revealed a strong positive correlation between the aldosterone level and the numbers of mineralocorticoid receptors: day $14-$ $\mathrm{R}=0.78,(\mathrm{p}=0.0022)$; day $21-\mathrm{R}=0.80,(\mathrm{p}=0.0018)$; day $35-\mathrm{R}=0.82$, $(p=0.0008)$.

Indicator values of the hepatocyte antioxidant system activity in rats of both groups in the early and late post-resuscitation period are demonstrated in Table 3. On day 1 after the IRP, a significant reduction in the activity of superoxide dismutase and catalase was observed in rats of the treatment group: by $50.3 \%$ and by $29 \%$, respectively $(p<0.0001)$. The lowest values of superoxide dismutase and catalase activity in the liver of treatment group rats were observed on days 3-7, with a further trend of recovery by day 35 , whereas in the control group, these indicators did not change significantly. Hence, rats unresistant to post-IRP hypoxia were characterized by a lower level of antioxidant protection.

\section{Discussion}

It is well-known that even a short period of ischemia in organs and tissues, followed by the restoration of blood flow, causes damage to tissues and cells, known as IPR injury [5]. In response to damage, hepatocytes produce cytokines, chemokines, and proteases, which contributes to the development of hepatocellular necrosis and apoptosis [8-11]. The liver is among the most sensitive to IRP organs [12]. Consequently, such interventions, as liver resection and transplantation, are accompanied by a pathological process of IRP injury [13]. Liver damage as a result of IRP may lead to the organ dysfunction, including graft rejection, which negatively affects the outcome of patient surgery and rehabilitation. Therefore, investigating the damage severity and timing of the post-IRP liver recovery remains a crucial issue of contemporary hepatology.

Rushing et al. showed that against the background of hemorrhagic shock in rats, occurring in response to IRP, the production of serum corticosterone is stimulated, which, in turn, triggers a cascade of processes: production of tumor necrosis factor (TNF- $\alpha$ ), release of cytokines and reactive oxygen species, etc. [14]. Stimulation of mineralocorticoid receptor expression in the liver against the background of IRP causes pro-inflammatory and profibrotic effects. It was discovered that an increase in the mRNA level in the liver significantly correlates with the expression of pro-inflammatory and profibrotic genes, while use of a specific mineralocorticoid receptor blocker, eplerenone, weakens these effects $[15,16]$. Our study has demonstrated that in rats, characterized by the low resistance to hypoxia, the vectors of fluctuations in the corticosterone concentrations, as well as in the numbers of its target receptors, were multidirectional on day 3 after the IRP: an increase in the corticosteroid level was accompanied by a reduction in the mineralocorticoid content. From the end of the second week of the post-resuscitation period, in the treatment group of animals, i.e., in those unresistant to hypoxia, the corticosterone level declined sharply while high aldosterone concentration in blood plasma was observed.

We have also established a strong direct statistically significant correlation between the aldosterone level and mineralocorticoid 
receptor content. Such pattern persisted until the end of the experiment, which allowed the aldosterone effects to manifest themselves. That is why, no significant increase in liver enzymes and TNF- $\alpha$ was observed in rats injected with aldosterone antagonists 30 minutes prior to the IRP simulation [17]. Therefore, we conclude that an increase in aldosterone after systemic IRP stimulates liver damage, while its antagonists have hepatoprotective properties.

Many authors pointed out the oxidative stress formation against the background of IRP. E.g., the study by Popov KA, et al. demonstrated high lability of the functional state of mitochondria with liver IRP [18]. The authors revealed a substantial decrease in the membrane potential of hepatocyte mitochondria after a 25minute compression of the liver vessels [18]. Kunak CS, et al reported a decrease in the activity of glutathione peroxidase and glutathione transferase after the liver IRP injury [19].

Our data suggested that rats, unresistant to post-IRP hypoxia, had a low level of antioxidant protection, compared with the control group. The lowest activity values of superoxide dismutase and catalase in the liver of rats were observed in the first week after the simulation of a systemic IRP. Therefore, it is during these particular periods that the body needs an antioxidant support.

To prevent and correct the oxidative stress, it is necessary to use antioxidant medications, e.g., vitamin $E$ and ascorbic acid, which have long been recognized as antioxidants [20]. The results of the study by Dossi CG, et al implied that in mice, treated with $\alpha$ tocopherol for 15 minutes before the simulation of liver IRP, mitochondrial function and superoxide dismutase activity did not decrease to critical levels, compared with the control group [8]. As stated by Cheung $E$, et al, zinc preparations reduce the degree of damage to hepatocytes under the IRP injury: 24 hours after the liver IRP, ALT and AST were 1.5-2 times lower in animals, treated with zinc the day before, compared with the control group $(p=0.025)[7]$.

Hence, liver injury and recovery against the background of IRP requires further investigation, particularly, studying the IRP pathogenesis and liver failure correction on long-term ischemic simulation models.

\section{Conclusion}

In animals, unresistant to hypoxia, systemic liver IRP was associated with pronounced changes in the dynamics of corticosteroid receptors, which led to high activity of the free radical oxidation and a reduction in the activity of key antioxidants. Starting from the second week, ischemia-reperfusion injury of the liver was accompanied by hypocorticosteronemia and hyperaldosteronemia, which created prerequisites for hyperstimulation of mineralocorticoid receptors. Therefore, the systemic ischemia reperfusion led to an oxidative damage to the liver, which required medicamentous correction in both early and late follow-up periods.

\section{Limitations}

Our study was limited by the sample size (number of rats) and duration of IRP simulation.

\section{Funding}

No external funding sources were used for this project.

\section{Conflict of interest}

Authors declare no conflicts of interest.

\section{References}

1. Cismen $\mathrm{O}$, Eken $\mathrm{H}$, Cimen FK, Cekic $\mathrm{AB}$, Kurt N, Bilgin $\mathrm{AO}$, et al. The effect of Liv-52 on liver ischemia reperfusion damage in rats. $B M C$ Pharmacol Toxicol 2020; 21(1): 2. https://doi.org/10.1186/s40360019-0380-0.

2. Kaprin AD, Sidorov DV, Rubtsova NA, Leontyev AV, Lozhkin MV, Petrov $L O$, et al. Prediction and prevention of liver failure after major liver primary and metastatic tumors resection. Research and Practical Medicine Journal 2016; 3(2): 13-21. Russian. https://doi.org/10.17709/2409-2231-2016-3-2-2.

3. Deng WS, $\mathrm{Xu} Q$, Liu YE, Jiang $\mathrm{CH}$, Zhou $\mathrm{H}$, Gu L. Effects of melatonin on liver function and lipid peroxidation in a rat model of hepatic ischemia/reperfusion injury. Exp Ther Med 2016; 11(5): 1955-1960. https://doi.org/10.3892/etm.2016.3160.

4. Dewar D, Moore FA, Moore EE, Balogh Z. Postinjury multiple organ $\begin{array}{llll}\text { failure. } \quad \text { Injury 2009; } & \text { 40(9): }\end{array}$ https://doi.org/10.1016/j.injury.2009.05.024.

5. Bajburina GA, Nurgaleeva EA, Shibkova DZ, Bashkatov SA. Method of determining degree of resistance to hypobaric hypoxia in small laboratory animals. Patent for invention No. 2563059. Russian. https://www.elibrary.ru/item.asp?id=37437741.

6. Myandina $\mathrm{Gl}$, Kulchenko NG, Alhejoj $\mathrm{H}$. The frequency of polymorphism -262 C>T CAT gene of infertile men in the Moscow region. Medical news of the North Caucasus 2019; 14(3): 478-481. https://doi.org/10.14300/mnnc.2019.14116.

7. Cheung E, Nikfarjam M, Jackett L, Bolton DM, Ischia J, Patel O. The protective effect of zinc against liver ischaemia reperfusion injury in a rat model of global ischaemia. J Clin Exp Hepatol 2020; 10(3): 228-235. https://doi.org/10.1016/j.jceh.2019.07.006.

8. Dossi CG, Vargas RG, Valenzuela R, Videla LA. Beneficial effects of natural compounds on experimental liver ischemia-reperfusion injury. Food Funct 2021; 12(9): 3787-3798. https://doi.org/10.1039/d1fo00289a.

9. Rozanova MV, Rozanov VE. The role of laser infrared therapy in treating traumatic liver lesions. Laser Medicine 2016; 20(3): 64 Russian. https://www.elibrary.ru/item.asp?id=27038764.

10. Roitberg GE, Sharkhun O. Management of patients with nonalcoholic fatty liver disease in the practice of a primary care physician. Journal of Family Medicine 2018; $\quad$ (5): $44-49$. https://www.elibrary.ru/item.asp?id=35590911.

11. Mendes-Braz M, Elias-Miró M, Jiménez-Castro MB, Casillas-Ramírez $A$, Ramalho FS, Peralta C. The current state of knowledge of hepatic ischemia-reperfusion injury based on its study in experimental models. J Biomed Biotechnol 2012; 2012: 298657. https://doi.org/10.1155/2012/298657.

12. Cannistrà $M$, Ruggiero $M$, Zullo $A$, Gallelli $G$, Serafini $S$, Maria $M$, et al. Hepatic ischemia reperfusion injury: A systematic review of literature and the role of current drugs and biomarkers. Int J Surg 2016; 33(Supp 1): S57-S70. https://doi.org/10.1016/j.ijsu.2016.05.050.

13. Nastos C, Kalimeris K, Papoutsidakis N, Tasoulis MK, Lykoudis PM, et al. Global consequences of liver ischemia/reperfusion injury. Oxid Med Cell Longev 2014; 2014 : 906965. https://doi.org/10.1155/2014/906965.

14. Rushing GD, Britt RC, Britt LD. Effects of hemorrhagic shock on adrenal response in a rat model. Ann Surg 2006; 243(5): 652-654. https://doi.org/10.1097/01.sla.0000216759.36819.1b.

15. Yamamoto K, Yamamoto T, Takamura M, Usui S, Murai H, Kaneko S, et al. Effects of mineralocorticoid receptor antagonists on responses to hemorrhagic shock in rats. World J Crit Care Med 2018; 7(1): 1-8. https://doi.org/10.5492/wjccm.v7.i1.1. 
16. Schreier B, Wolf A, Hammer S, Pohl S, Mildenberger S, Rabe S, et al. The selective mineralocorticoid receptor antagonist eplerenone prevents decompensation of the liver in cirrhosis. Br J Pharmacol 2018; 175(14): 2956-2967. https://doi.org/10.1111/bph.14341.

17. Atalay S, Soylu B, Aykac A, Ogunc AV, Cetinel S, Ozkan N, et al. Protective effects of spironolactone against hepatic ischemia/reperfusion injury in rats. Turk J Surg 2019; 35(4): 285-292. https://doi.org/10.5578/turkjsurg.4340.

18. Popov KA., Bykov IM, Tsymbalyuk IYu, Kurzanov AN, Storozhuk AP, Esaulenko EE. Changes in the membrane potential of mitochondria in dependence on the continuance of ischemia and reperfusion of liver in rats. Medical news of the North Caucasus 2019; 14(1.2): 231-234. Russian https://doi.org/10.14300/mnnc.2019.14022.

19. Kunak CS, Kukula O, Mutlu E, Genc F, Peker GG, Kuyrukluyildiz U, et al. The effect of Etoricoxib on hepatic ischemia-reperfusion injury in rats. Oxid Med Cell Longev 2015; 2015: 598162. https://doi.org/10.1155/2015/598162.

20. Kulchenko NG. Antioxidant therapy for patospermia. Bulletin of the Medical Institute "REAVIZ" (Rehabilitation, Doctor and Health). 2018; (1(31)): 41-48. Russian https://elibrary.ru/item.asp?id=32823966.

Authors:

Gulnar A. Bayburina - PhD, Assistant Professor, Department of Physiological Pathology, Bashkir State Medical University, Ufa, Russia. https://orcid.org/0000-0002-4741-6295.

Elena A. Nurgaleeva - DSc, Professor, Department of Physiological Pathology, Bashkir State Medical University, Ufa, Russia. https://orcid.org/0000-0003-1523-4566.

Ajgul F. Samigullina - PhD, Assistant Professor, Department of Physiological Pathology, Bashkir State Medical University, Ufa, Russia. https://orcid.org/0000-0001-9026-7259.

Ekaterina R. Farshatova - DSc, Professor, Department of Physiological Pathology, Bashkir State Medical University, Ufa, Russia. https://orcid.org/0000-0003-3790-72566.

Timur I. Ganeev - PhD, Associate Professor, Department of Prosthetic Dentistry and Maxillofacial Surgery, Bashkir State Medical University, Ufa, Russia. https://orcid.org/0000-0001-9716-3380.

Eduard F. Agletdinov - DSc, Professor, Department of Physiological Pathology, Bashkir State Medical University, Ufa, Russia. https://orcid.org/0000-0002-6256-2020.

Tatyana V. Tarasova - DSc, Professor, Chair of the Department of Normal Physiology and Physiological Pathology, Institute of Medicine, Ogarev State University of Mordovia. Saransk, Russia. http://orcid.org/0000-0001-9745$\underline{9739}$ 